\title{
Structure of dislocations in Al and Fe as studied by positron-annihilation spectroscopy
}

Hidalgo, C.; González-Doncel, G.; Linderoth, Søren; San Juan, J.

\section{Published in:}

Physical Review B

Link to article, DOI:

10.1103/PhysRevB.45.7017

Publication date:

1992

Document Version

Publisher's PDF, also known as Version of record

Link back to DTU Orbit

Citation (APA):

Hidalgo, C., González-Doncel, G., Linderoth, S., \& San Juan, J. (1992). Structure of dislocations in Al and Fe as studied by positron-annihilation spectroscopy. Physical Review B, 45(13), 7017-7021.

https://doi.org/10.1103/PhysRevB.45.7017

\section{General rights}

Copyright and moral rights for the publications made accessible in the public portal are retained by the authors and/or other copyright owners and it is a condition of accessing publications that users recognise and abide by the legal requirements associated with these rights.

- Users may download and print one copy of any publication from the public portal for the purpose of private study or research.

- You may not further distribute the material or use it for any profit-making activity or commercial gain

- You may freely distribute the URL identifying the publication in the public portal 


\title{
Structure of dislocations in $\mathrm{Al}$ and $\mathrm{Fe}$ as studied by positron-annihilation spectroscopy
}

\author{
C. Hidalgo \\ Asociación EURATOM/CIEMAT, Avenida Complutense 22, 28040 Madrid, Spain \\ G. González-Doncel \\ Centro Nacional de Investigaciones Metalúrgicas, Consejo Superior de Investigaciones Científicas, \\ Avenida de Gregorio del Amo 8, 28040 Madrid, Spain \\ S. Linderoth \\ Laboratory of Applied Physics, Technical University of Denmark, 2800 Lyngby, Denmark \\ J. San Juan \\ Departamento de Física de la Materia Condensada, Facultad de Ciencias, Universidad del País Vasco, \\ Apartado 644, 48080 Bilbao, Spain
}

(Received 22 March 1991)

\begin{abstract}
Positron-lifetime-spectra measurements have been performed on single crystals of $\mathrm{Al}$ and polycrystals of Fe deformed under the tensile mode at room temperature and $77 \mathrm{~K}$, respectively. It is shown that the positron-trapping component in $\mathrm{Al}$ depends on the number of slip systems activated: The positron lifetime is 215,220 , and 240 ps when single-, double-, and multiple-slip planes are activated, respectively. This dependence is well understood if positrons annihilate at dislocation-associated defects (jogs) with different positron-annihilation characteristics revealing different jog structures. In iron, the positronlifetime spectra associated with dislocations were found to be independent of the annealing temperature between 110 and $360 \mathrm{~K}$. In this temperature range, screw dislocations should transform into nonscrew dislocations, suggesting that the positrons are not sensitive to the different types of dislocations. These results are interpreted on the basis that positrons annihilate at defects associated with the dislocations rather than at the dislocation lines.
\end{abstract}

\section{INTRODUCTION}

Positron-annihilation spectroscopy is a useful technique to study defects in materials. ${ }^{1,2}$ The most straightforward studies can be performed on open-structure defects in metals such as vacancies or voids. For example, the positron lifetime in monovacancies is about $50 \%$ longer than in the perfect lattice. ${ }^{3}$ This lifetime increases further for the large three-dimensional vacancy agglomerations. The positron lifetime in large voids without contaminations is about 500 ps, much longer than in the perfect lattice. ${ }^{4,5}$

Positrons are also sensitive to dislocations. In fact, evidence for positron trapping and annihilation at defects was, indeed, found for samples into which dislocations were induced. ${ }^{6,7}$ A large number of positron annihilation studies have dealt with the interaction of positrons with dislocations in metals. ${ }^{8-14}$ It has been found that the positron lifetime associated with dislocations is close to that at monovacancies. However, the identity of the sites of the dislocation at which the positrons trap and annihilate is still under debate. It has been proposed that positrons can either annihilate at the dislocation line or at defects associated with the dislocation, such as jogs. Experiments carried out on deformed Zn (Ref. 10) and Cd (Ref. 13) have shown that the positron-dislocation interaction can be well described assuming that positrons are trapped at dislocation lines where they are either trapped into de- fects associated with the dislocation (jogs) and annihilate, or detrap back into the perfect lattice. ${ }^{15-17}$ From positron-lifetime measurements in deformed $\mathrm{Zn},{ }^{14}$ it was deduced that the positron binding energy was around 40 $\mathrm{meV}$. It has also been proposed that positrons are sensitive to the length and structure of the jogs. ${ }^{10,13}$

In this paper, further studies of the influence of the jog structure on the positron-annihilation characteristics are presented. The goal of the present paper is twofold: first, to study the influence of the number of slip systems activated during deformation in $\mathrm{Al}$ on the positron-lifetime spectra, and second, to study the influence of screw and edge dislocations in $\mathrm{Fe}$ on the positron-lifetime parameters.

\section{EXPERIMENT}

Aluminum single crystals ( $>99.8 \%$ purity) were grown from 7-mm-diam extruded rods by a strain anneal method as described elsewhere. ${ }^{18}$ Rods were $330 \mathrm{~mm}$ maximum length, and three tensile samples from each single crystal (i.e., with the same initial orientation) could be obtained. The crystalline orientations of the rod axis were determined by the Laue method. Cylindrical samples for tensile tests were machined from the singlecrystal rods $30 \mathrm{~mm}$ in length and $5 \mathrm{~mm}$ in diameter. In order to remove damage introduced during machining the samples were annealed at $625 \mathrm{~K}$ for $20 \mathrm{~min}$ and then 
furnace cooled.

In Fig. 1 the orientations of the single crystals chosen for this investigation are shown. The orientation labeled SINGLE SLIP is distant from the symmetry boundary [001]-[111] and corresponds to tensile samples that deform extensively by slip only on the primary system (111)[101]. For these samples, $56 \%$ elongation by single slip on the primary system was needed to activate the conjugate slip system $(\overline{1} 1)[\overline{1} 01]$. On the other hand, the orientation labeled DOUBLE SLIP is close to the sym-

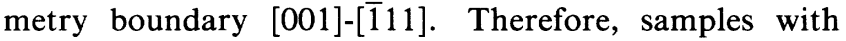
this orientation deformed by the simultaneous activation of both the primary and the conjugate slip systems almost from the early stage of deformation. For these samples, only $2.7 \%$ elongation by slip on the primary system was required to activate the conjugate slip system.

Aluminum samples of both orientations (single and double slip) were strained $8 \%, 16 \%$ and $27 \%$ at room temperature at an initial strain rate of $\dot{\varepsilon}_{0}=1.1 \times 10^{3} \mathrm{~s}^{-1}$ on a conventional tensile testing machine. As mentioned in the above paragraph these elongations are, for samples deformed by single slip, well below the elongation needed to activate the conjugate slip system. For samples deformed by double slip these elongations are, however, high enough to guarantee simultaneous activation of both the primary and conjugate slip systems during deformation. After straining, samples were removed from the tensile machine before any necking would appear. A uniform deformation in the gauge length of samples was thereby produced.

In order to activate more than two slip systems, a set of aluminum samples were deformed by rolling (20\% reduction) at room temperature. For comparison, wellannealed Al (99.9999\% purity) specimens were, similarly, deformed by rolling.

Specimens for positron-annihilation measurements were prepared in the form of 2-mm-thick discs that were cut from the gauge length of the tested samples. For this purpose a $\mathrm{SiC}$ wheel working at a low cutting speed using methanol as a lubricant was utilized. Superficial oxide and damage caused by the cutting was removed from the

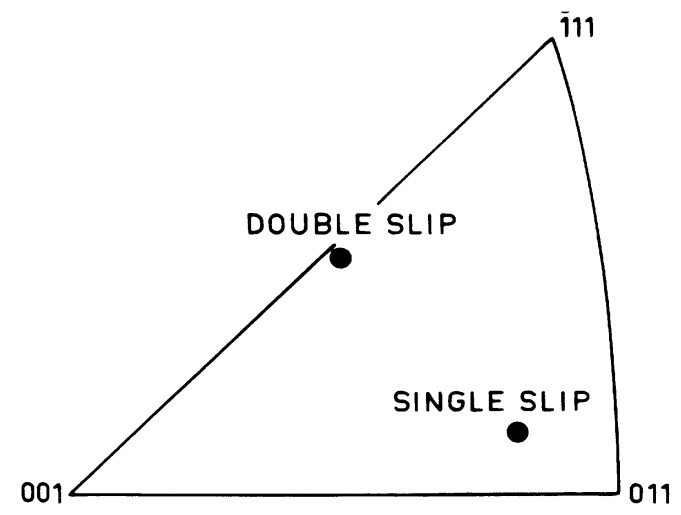

FIG. 1. Stereographic triangle showing the orientation of both types of tensile specimens: (a) single slip (the tensile axis is distant from the symmetry boundary [001]-[1111]), (b) double slip (the tensile axis is on the vicinity of the symmetry boundary). surfaces to be exposed to the positron source by electropolishing in a solution of two parts of methanol and one part of nitric acid at $250 \mathrm{~K}$.

Polycrystalline iron $(99.998 \%$ purity) samples purified by a zone melting technique were supplied by Centre d'Etudes Nucléaires at Grenoble. ${ }^{19}$ The initial ingot was laminated to a final thickness of $0.5 \mathrm{~mm}$, and samples $\left(50 \times 5 \times 5.5 \mathrm{~mm}^{3}\right)$ for tensile tests were cut and annealed in a dry hydrogen atmosphere at $675 \mathrm{~K}$ for $10 \mathrm{~h}$. The final microstructure observed by optical and transmission-electron microscopy ${ }^{19,20}$ showed an equiaxial grain size of $100 \mathrm{~mm}$ with a low dislocation density. In order to avoid twinning during deformation at low temperature, ${ }^{19}$ samples were deformed $5 \%$ at room temperature. In this manner, mobile dislocations are created. These mobile dislocations will generate further strain when samples are again pulled at low temperature. As edge dislocations are mobile at $77 \mathrm{~K}$, samples deformed $6 \%$ at this temperature would induce, primarily, screw dislocations. ${ }^{20,21}$ In order to freeze the screw dislocation structure, tensile specimens were maintained at low temperature $(77 \mathrm{~K})$ for the positron-annihilation measurements.

Positron-lifetime measurements were performed by using a conventional fast-slow spectrometer with a resolution of $260 \mathrm{ps}$ (full width at half maximum). $\mathrm{A}^{22} \mathrm{Na}$ positron source was evaporated onto a thin $\mathrm{Ni}$ foil $(0.45$ $\mathrm{mg} / \mathrm{cm}^{2}$ ). The fraction of positron annihilating in the nickel foil has been estimated to be about $4 \% .^{22}$

The component due to annihilation in the salt was obtained from positron-lifetime spectra acquired at room temperature on well-annealed aluminum in which only one component, due to free annihilation in the bulk, is expected. After foil correction, the spectra could be decomposed into two lifetime components: the short one $\left(\tau_{f} \approx 162 \mathrm{ps}\right)$, due to the free annihilation in the bulk, and the long one $\left(\tau_{f} \approx 450 \mathrm{ps}\right)$, with intensity about $3 \%$, due to annihilation in the positron source.

Aluminum samples were studied by positron-lifetime measurements at room temperature. The samples elongated $16 \%$ (both single and double slip) were also studied in the temperature range $12-300 \mathrm{~K}$. Iron samples were studied with positron-lifetime measurements acquired at $100 \mathrm{~K}$ after isochronal annealings of $30 \mathrm{~min}$ between 100 and $360 \mathrm{~K}$.

\section{RESULTS}

\section{A. Aluminum}

Figure 2 shows a stress-strain plot for the two differently oriented single crystals strained $27 \%$. As expected, the tensile sample oriented for double slip deformed under higher flow stress than the sample oriented for single slip. This difference is due to the interaction between dislocations of two different slip systems which is present in the sample deformed by double slip, but negligible in the sample deformed by single slip.

Satisfactory fits of the lifetime spectra were obtained for all aluminum samples by using a two-component decomposition. From the two trapping components 


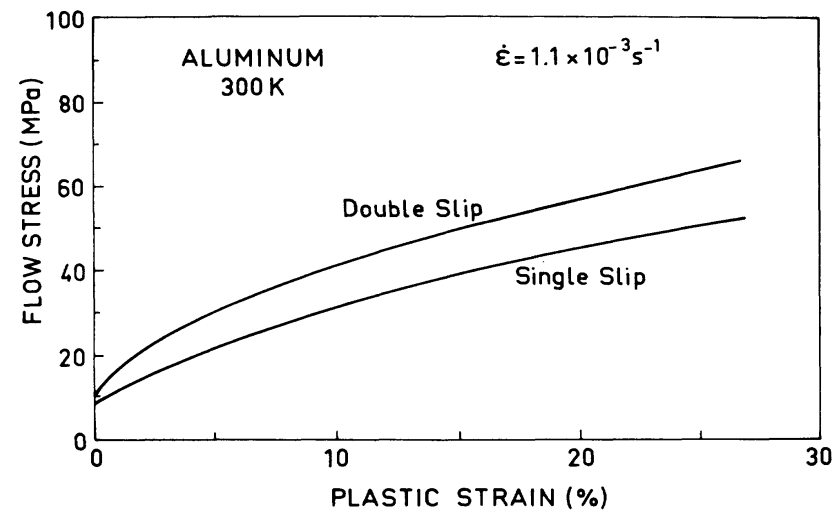

FIG. 2. Stress-strain curves at room temperature for aluminum single crystals initially oriented for single and double slip.

$\left(\tau_{1}, \tau_{2}\right)$ and the corresponding intensities $\left(I_{1}, I_{2}\right)$ the mean positron lifetime $\left(\bar{\tau}=I_{1} \tau_{1}+I_{2} \tau_{2}\right)$ has been calculated. For Al deformed by single and double slip the long-lived trapping component $\left(\tau_{2}\right)$ was 215 and $220 \pm 2$ ps, respectively. In contrast, for samples deformed by cold rolling (i.e., multiple slip) $\tau_{2}$ was found to be $240 \mathrm{ps}$ in both the $99.8 \%$ and $6 \mathrm{~N}$ purity samples.

Figure 3 shows the evolution of the mean positron lifetime measured at $300 \mathrm{~K}$ as a function of elongation for $\mathrm{Al}$ samples deformed by single and double slip. It can be seen that the positron mean lifetime increases with increasing deformation. This result implies that the fraction of positrons annihilating at defects induced by deformation increases with increasing elongation. The difference in positron lifetime between single and double slip can be well appreciated for elongations in excess of $10 \%$. In order to examine thoroughly the origin of this difference, a more detailed study for samples deformed $16 \%$ was carried out.

Figures 4 and 5 show the temperature dependence of the positron-annihilation parameters for $\mathrm{Al}$ deformed $16 \%$ in the tensile mode where one- and two-slip systems,

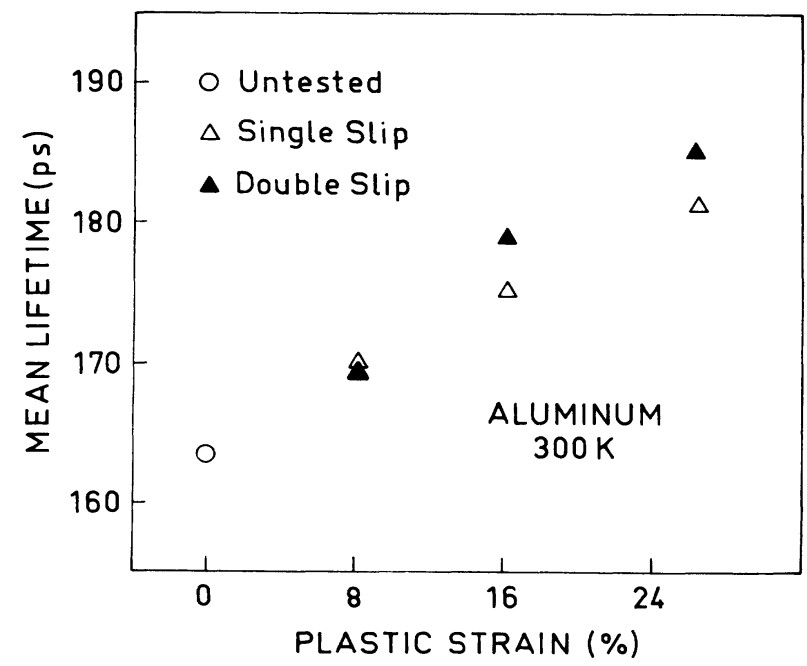

FIG. 3. Mean positron lifetime at room temperature as a function of plastic strain for aluminum single crystals deformed by single and double slip.

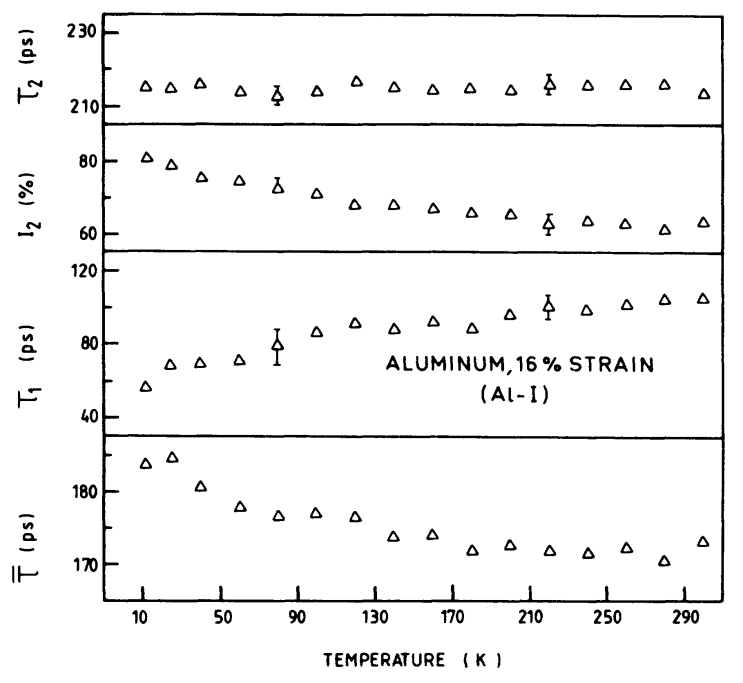

FIG. 4. Positron-annihilation parameters as a function of temperature for aluminum deformed by single slip.

respectively, were activated. It is to be noted that the positron lifetime of the trapping component, $\tau_{2}$, is about 215 ps for single slip and 220 ps for double slip, respectively, and did not alter with measuring temperature. These values are clearly shorter than the $240 \mathrm{ps}$ observed for the samples deformed by rolling (multiple slip) and the $245-250$ ps observed for positron annihilation from monovacancies in aluminum. ${ }^{23,24}$

The one-trap model was found to describe reasonably well the positron-lifetime spectra in the temperature range studied in this investigation. The trapping rate $K$ may, therefore, be obtained from the following formula:

$$
K=\left(1-\bar{\tau} \lambda_{f}\right) /\left(\bar{\tau}-\tau_{2}\right),
$$

where $\lambda_{f}\left(\equiv 1 / \tau_{f}\right)$ is the positron-annihilation rate in the perfect lattice $\left(\tau_{f} \approx 163 \mathrm{ps}\right)$.

The temperature dependence of the positron trapping rate is shown in Fig. 6. In the temperature range 12-120

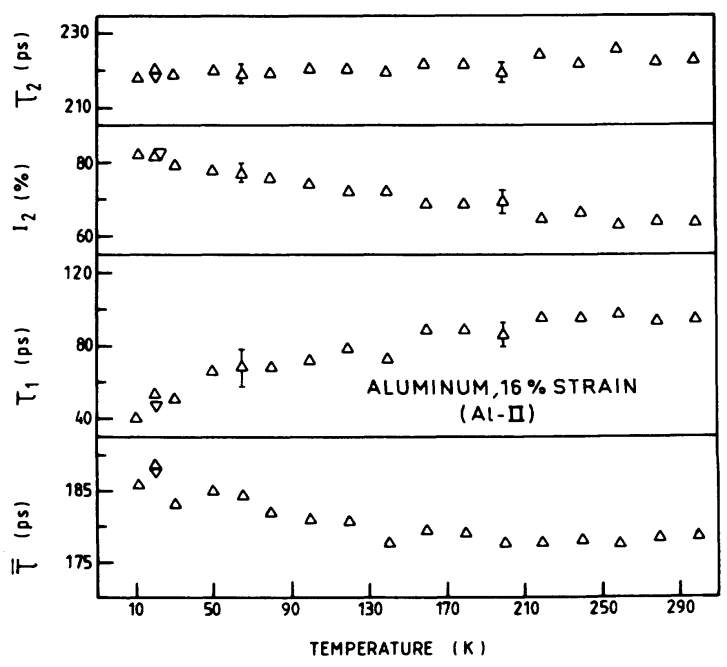

FIG. 5. Positron-annihilation parameters as a function of temperature for aluminum deformed by double slip. 


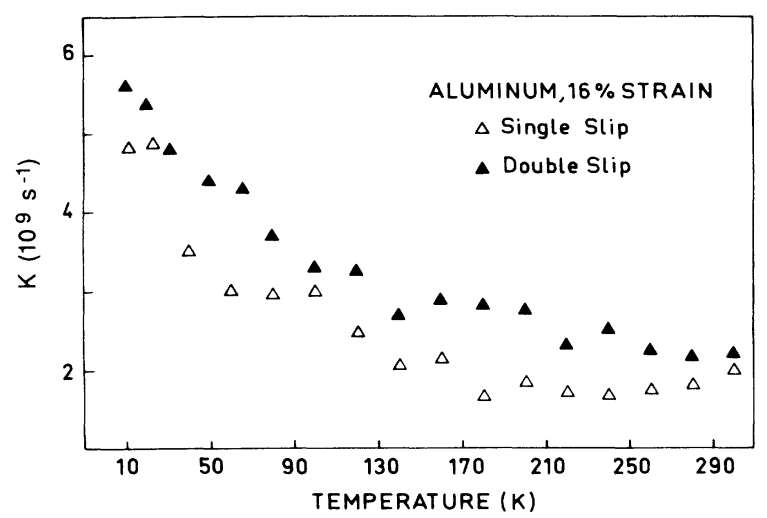

FIG. 6. Trapping rate as a function of temperature for aluminum single crystals deformed $16 \%$ by single and double slip.

$\mathrm{K}$ the trapping rate decreases with increasing temperature. Above $180 \mathrm{~K}$, the trapping rate is basically irrespective of temperature.

\section{B. Iron}

The positron-lifetime spectra were well fitted with two components with the trapping component having a positron lifetime of $150 \pm 4$ ps. This value is significantly shorter than that assigned to annihilation from vacancies $(175 \mathrm{ps}){ }^{25}$ and we attribute this component to positron annihilation from dislocations. The temperature dependence of the positron-lifetime parameters are shown in Fig. 7. The positron-lifetime spectra were found not to change upon annealing in the temperature range $100-360 \mathrm{~K}$.

\section{DISCUSSION}

\section{A. Aluminum}

When metals are plastically deformed at temperatures at which vacancies are mobile, three-dimensional vacancy

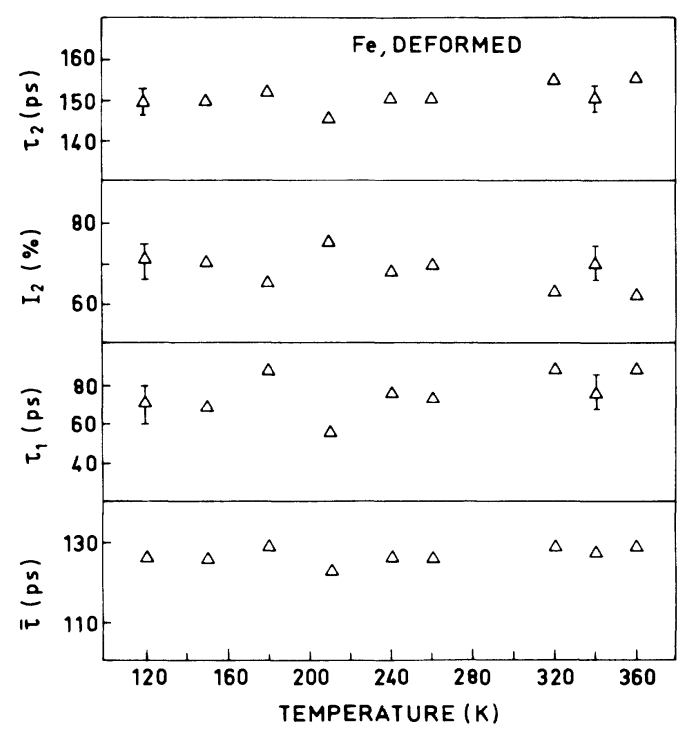

FIG. 7. Positron-annihilation parameters as a function of temperature for iron deformed at $77 \mathrm{~K}$. agglomerates, besides dislocations, may be formed. Positrons are very sensitive to the size of the open volume of defects. Positron lifetime increases with increasing of the open-volume size of the defects. ${ }^{26}$ The testing temperature of $300 \mathrm{~K}$ is well above that for vacancy migration in aluminum $(\approx 230 \mathrm{~K}){ }^{4}$ On the other hand, the positron lifetime of the trapping component in our tensile strained aluminum single crystals was found to be $215-220$ ps, which is even well below the positron lifetime in monovacancies. This suggests that if three-dimensional clusters were formed during the deformation process, their concentration had to be under the detection limit for the positrons. Hence, we conclude that the positron-trapping component originates from positron trapping and annihilation at dislocations.

An ordinary rolling deformation of the single-crystal material, as well as rolling of a $99.9999 \%$-purity Al sample set, yielded a positron lifetime of $240 \mathrm{ps}$ for the trapped positrons. This value is in agreement with those previously quoted for dislocations in aluminum generated either by cold work ${ }^{4,8}$ or neutron irradiation. ${ }^{27}$ This value is also close to the experimentally determined positron lifetime in monovacancies of aluminum (245-250 ps). ${ }^{23,24}$ The positron lifetimes of $215-220 \mathrm{ps}$, which we attribute to positron annihilation at dislocations generated by single or double slip, are 20-25 ps shorter than that obtained by rolling. As can be seen in Figs. 4 and 5, the values of 215 and 220 ps remain constant in the temperature range $12-300 \mathrm{~K}$. This indicates that these positron lifetimes are not merely a mixture of several components. It can, therefore, be inferred that the 215- and 220-ps components are, indeed, due to positron annihilation from sites associated with dislocations, but that these sites differ in nature from those associated with dislocations produced by cold work or neutron irradiation.

Positron-lifetime investigations during the annealing process in deformed $\mathrm{Zn}$ (Ref. 10) and Cd (Ref. 8) indicate that positrons are sensitive to the structure of jogs. The results for $\mathrm{Zn}$ and $\mathrm{Cd}$ were interpreted as positron annihilation at long (multiple) jogs and single jogs. ${ }^{10,13}$ The lifetimes of 215,220 , and 240 ps associated with the trapping component of positrons in deformed aluminum may, likewise, be assigned to positron annihilation at jogs, where the different mean lifetime is attributed to different structures of the jogs. In the case of deformation by single slip the intersection between dislocation lines is negligible and it can be assumed that most jogs are generated by the presence of the Peierls energy, i.e., the jogs are in the same slip plane (kink). In the case of activation of two slip planes, jogs are generated by the intersection of dislocation lines from different slip systems (i.e., jogs are on planes that are different from the slip plane). When deformation is caused by rolling, many slip systems are activated and the jogs associated with dislocations are now generated by a complex intersection of dislocation lines. Possibly, the jogs are extended jogs (multiple jogs) ${ }^{10,13}$ or vacancies associated with the dislocations.

The temperature dependence of the positron-trapping rate is similar to that observed for dislocations loops in $\mathrm{Al}$ (Ref. 27) and Mo. ${ }^{9}$ The trapping rate decreases rapidly with increasing temperature from 10 to $120 \mathrm{~K}$. At 
high temperatures, the trapping rate is basically constant. A similar temperature dependence has been observed in Zn (Ref. 14) below $120 \mathrm{~K}$, whereas above $120 \mathrm{~K}$ the temperature dependence was interpreted in terms of thermally activated positron detraping from the dislocation lines. This implies a positron-dislocation binding energy of about $40 \mathrm{meV} .^{14}$ The absence of a temperature dependence above $120 \mathrm{~K}$ in deformed $\mathrm{Al}$ suggests that the positron-dislocation binding energy is above approximately $0.1 \mathrm{eV}$, in agreement with recent theoretical calculations by Häkkinen, Mäkinen, and Manninen. ${ }^{28}$

The conclusion that positrons trapped and annihilated at single jogs produced either by single or double slip have lifetimes significantly below that at monovacancies, is supported by the calculations of Häkkinen, Mäkinen, and Manninen. ${ }^{28}$ These authors found that positrons trapped at vacancies associated with dislocations and at single jogs may result in lifetimes about 55 ps longer than in the bulk. We observe lifetimes that are 50-55 ps longer than in the bulk, which reveals a good agreement with their calculations.

\section{B. Iron}

It is expected that mainly screw dislocations are produced when iron samples are deformed at $77 \mathrm{~K}$ in the tensile mode. Screw dislocations become unstable above $240 \mathrm{~K}$, thereby increasing the relative concentration of nonscrew dislocations. ${ }^{20,21}$ The lack of any measurable dependence of the positron-lifetime components on the annealing temperature shows that, within our experimen- tal error, positrons cannot distinguish between nonscrew and screw dislocations. This conclusion is seemingly in contradiction with previous experiments ${ }^{11}$ where positron lifetimes of 142 and 165 ps were assigned for positron annihilation at screw and edge dislocations, respectively. However, the present results in iron are consistent with those obtained in aluminum in this investigation (i.e., with the model in which positrons are finally annihilated at jogs rather than at the dislocation line).

\section{CONCLUSIONS}

Positron-lifetime measurements carried out on Al deformed by single, double, and multiple slip and iron deformed by multiple slip at $77 \mathrm{~K}$ gave the following conclusions: (i) The positron lifetime associated with dislocations in aluminum depends on the number of slip systems activated. The lifetime increases with an increasing number of slip systems. (ii) This difference is interpreted in terms of positron annihilation at jogs with different structures (relaxations). (iii) In Fe deformed at $77 \mathrm{~K}$, positrons are found not to be able to distinguish between nonscrew and screw dislocations.

\section{ACKNOWLEDGMENTS}

Two of the authors (G.G.-D. and J.S.J.) wish to acknowledge partial support to this work provided by CICYT (Comisión Interministerial de Ciencia y Tecnología, Spain) under project No. MAT89-0554-C02.
${ }^{1}$ Positrons in Solids, edited by P. Hautojärvi (Springer-Verlag, Berlin, 1979).

${ }^{2}$ Positron Solid State Physics, edited by W. Brandt and A. Dupasquier (North-Holland, Amsterdam, 1983).

${ }^{3}$ R. Nieminen, in Positron Solid State Physics (Ref. 2), p. 359.

${ }^{4}$ K. Petersen, in Positron Solid State Physics (Ref. 2), p. 197.

${ }^{5}$ P. Hautojärvi, J. Heiniö, M. Manninen, and R. Nieminen, Philos. Mag. 35, 973 (1977).

${ }^{6}$ I. Y. Dekhyar, D. A. Levina, and V. S. Mikhalenkov, Dokl. Akad. Nauk SSSR 156, 795 (1964) [Sov. Phys. Dokl. 9, 494 (1964)].

${ }^{7}$ S. Berko and J. C. Erskine, Phys. Rev. Lett. 19, 309 (1967).

${ }^{8}$ P. Hautojärvi, A. Tamminen, and P. Janho, Phys. Rev. Lett. 24, 459 (1970).

${ }^{9}$ M. D. Bentzon, S. Linderoth, and K. Petersen, in Positron Annihilation, edited by P. C. Jain, R. M. Singru, and K. P. Gopinathan (World Scientific, Singapore, 1985), p. 485.

${ }^{10}$ C. Hidalgo, S. Linderoth, and N. de Diego, Philos. Mag. Lett. A 54, L61 (1986).

${ }^{11}$ Y. K. Park, J. T. Waber, M. Meshii, Jr., C. L. Snead, and C. G. Park, Phys. Rev. B 34, 823 (1986).

${ }^{12}$ S. Linderoth and C. Hidalgo, Phys. Rev. B 6, 4054 (1987).

${ }^{13}$ C. Hidalgo and S. Linderoth, J. Phys. F 18, L263 (1988).

${ }^{14}$ C. Hidalgo, S. Linderoth, and N. de Diego, Phys. Rev. B 36, 6740 (1987).

15J. W. Martin and R. Paetsch, J. Phys. F 2, 997 (1972).

${ }^{16}$ M. Doyama and R. M. J. Cotterill, in Positron Annihilation, edited by R. R. Hasiguti and K. Fujiwara (Japan Institute of Metals, Sendai, 1979), p. 89.

${ }^{17}$ L. C. Smedskjaer, M. Manninen, and M. J. Fluss, J. Phys. F 10, 2237 (1980).

${ }^{18} \mathrm{~K}$. T. Aust, The Art and Science of Growing Crystals (Wiley, New York, 1963).

${ }^{19} \mathrm{~F}$. Vanoni, Ph.D. dissertation, University Scientifique et Médical, Grenoble, 1973.

20J. San Juan, Ph.D. dissertation, Universidad del País Vasco, Bilbao, 1985.

${ }^{21}$ L. P. Kuvin, Rev. Deform. Behav. Mater. 1, 244 (1976).

${ }^{22}$ S. Linderoth, H. E. Hansen, B. Nielsen, and K. Petersen, Appl. Phys. A 33, 25 (1984).

${ }^{23}$ H. E. Schaefer, R. Gugelmeier, R. Schmolz, and A. Seeger, in Microstructural Characterization of Materials by NonMicroscopical Techniques, edited by N. H. Andersen et al. (Riso National Laboratory Roskilde, 1984), p. 489.

${ }^{24}$ H. Rajainmäki and S. Linderoth, J. Phys. Condens. Matter 2, 6623 (1990).

${ }^{25}$ G. Kuramoto, Y. Asano, M. Takanaka, and K. Kitajimi, J. Phys. Soc. Jpn. 53, 1098 (1983).

${ }^{26}$ P. Hautojärvi, J. Heiniö, M. Manninen, and R. Nieminen, Philos. Mag. 35, 973 (1977).

${ }^{27}$ S. Linderoth, M. D. Bentzon, H. E. Hansen, and K. Petersen, in "Positron Annihilation" (Ref. 9), p. 494.

${ }^{28}$ H. Häkkinen, S. Mäkinen, and M. Manninen, Europhys. Lett. 9, 809 (1989). 